Disponível em

http://www.anpad.org.br/rac

RAC, Rio de Janeiro, v. 17, n. 4, art. 6, pp. 499-517, Jul./Ago. 2013

$(\mathrm{coc})$ EY-NG

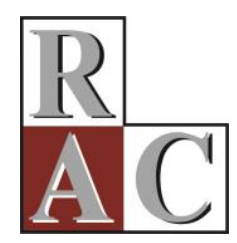

\title{
Cognição e Sustentabilidade: Estudo de Casos Múltiplos no Índice de Sustentabilidade Empresarial da BM\&FBovespa
}

\section{Cognition and Sustainability: Multiple Case Studies of the BM\&FBovespa Corporate Sustainability Index}

Farley Simon Nobre

E-mail: fsmnobre@gmail.com

Universidade Federal do Paraná - PPGADM/UFPR UFPR, Av. Pref. Lothario Meissner, 632, $2^{\circ}$ andar, Jardim Botânico, 80210-170, Curitiba, PR, Brasil.

Rhubens Ewald Moura Ribeiro E-mail: rhubens7@yahoo.com.br Universidade Federal do Paraná - PPGADM/UFPR UFPR, Av. Pref. Lothario Meissner, 632, $2^{\circ}$ andar, Jardim Botânico, 80210-170, Curitiba, PR, Brasil. 


\title{
Resumo
}

A busca da sustentabilidade nas organizações demanda de seus participantes uma complexidade cognitiva mais elevada devido às suas dimensões sistêmicas e aos seus múltiplos critérios associados à economia, à sociedade $\mathrm{e}$ ao meio ambiente. Este artigo estuda a relação entre Grau de Complexidade Cognitiva (GCC) e Sustentabilidade em Organizações (SEO) de empresas listadas no Índice de Sustentabilidade Empresarial (ISE) da Bolsa de Valores de São Paulo (BM\&FBOVESPA). Como metodologia de pesquisa, adotou-se uma abordagem qualitativa, conduzida por meio de estudo de casos múltiplos. Utilizou-se pesquisa documental para levantar relatórios de sustentabilidade e questionários do ISE que foram submetidos à análise de conteúdo. A amostra intencional foi constituída por seis empresas que disponibilizaram por completo seus relatórios de sustentabilidade e questionários do ISE. As avaliações de GCC e SEO das empresas foram realizadas respectivamente sob as óticas do modelo de Criação de Valor Sustentável (CVS) e das dimensões, critérios e indicadores do ISE. Resultados de análises permitiram verificar similaridades e diferenças entre os casos estudados e confirmaram a existência de uma relação com tendência positiva entre GCC e SEO.

Palavras-chave: cognição; mapas cognitivos; sustentabilidade; valor sustentável; ISE (BM\&FBOVESPA).

\begin{abstract}
The search for organizational sustainability in organizations demands a higher cognitive complexity from their participants due to their systemic dimensions and various aspects associated with the economy, society and environment. This paper studies the relationship between Degree of Cognitive Complexity (DCC) and Sustainability in Organizations (SIO) of companies that participate in the Corporate Sustainability Index (CSI) of Bolsa de Valores of São Paulo (BM \& FBOVESPA - the Brazilian Stock Exchange). Its research methodology was based on a qualitative approach and multiple case studies. Document research was used to find sustainability reports and CSI questionnaires were implemented and then subjected to content analysis. An intentional sample was composed of six companies that made their full sustainability reports and CSI questionnaires available. The companies' DCC and SIO were respectively evaluated from the perspectives of Sustainable Value Creation (SVC) and Corporate Sustainability Index (CSI). Comparative analyses showed similarities and differences among the cases and a tendency for a positive relationship between DCC and SIO.
\end{abstract}

Key words: cognition; cognitive maps; sustainability; sustainable value; ISE (BM\&FBOVESPA). 


\section{Introdução}

Coletivamente, o meio ambiente e a sociedade formam um ecossistema (Beeby \& Brennan, 2007) que tem sido gradativamente influenciado pelo homem e seus diversos agrupamentos sociais por meio de decisões. Essas decisões são tomadas em ambientes situacionais complexos, e a relevância das suas implicações sociais e ambientais depende do grau de complexidade cognitiva dos seus decisores (Maia \& Pires, 2011; Nobre, Tobias, \& Walker, 2010, 2011). No sistema capitalista e em seus modelos de produção, as decisões se mostram pautadas na maximização ou na satisfação do desempenho econômico. Sendo assim, as organizações têm explorado os recursos naturais de maneira intensa, desordenada e insensata, além de escalonarem uma distribuição desequilibrada desses recursos, agravando problemas complexos relativos à degradação do meio ambiente, à mudança climática, aos conflitos culturais, à redução da moral, à injustiça social, entre outros (Thompson, 2010; United Nations Environment Programme [UNEP], 2012; Organização das Nações Unidas para a Educação, a Ciência e a Cultura [UNESCO], 2005; United Nations World Commission on Environment and Development [WCED], 1987). Surge, então, a necessidade de reflexão sobre a proposição de um desenvolvimento alternativo ao crescimento econômico, como resultado da má qualidade na relação entre homem, organização, sociedade e natureza. Entre esses elementos, a organização, e, particularmente, as empresas são objetos de estudo neste artigo.

Entre as premissas deste trabalho, destaca-se que os participantes das organizações, especialmente executivos e acionistas, podem interpretar a sustentabilidade como importante fonte de criação de valor que permeia as estratégias e as operações da organização. Decisões baseadas nessa interpretação poderão proporcionar benefícios econômicos, sociais e ambientais baseados no triple bottom line (Elkington, 1994, 1997; Hart, 2005; Hart \& Milstein, 2003) e contribuir para um desenvolvimento sustentável. Por ser um tema interdisciplinar em constante evolução, e que leva em consideração dimensões sistêmicas e múltiplos critérios associados à economia, à sociedade e ao meio ambiente, a sustentabilidade demanda uma complexidade cognitiva mais elevada dos participantes nas organizações.

Diante desse contexto, este artigo contribui ao investigar a relação entre Grau de Complexidade Cognitiva (GCC) e Sustentabilidade em Organizações (SEO) de empresas que participam no Índice de Sustentabilidade Empresarial (ISE) da Bolsa de Valores de São Paulo (BM\&FBOVESPA). Espera-se, ainda, que os resultados encontrados possam contribuir para o direcionamento de trabalhos futuros sobre estudos contingenciais entre as categorias analisadas (GCC e SEO). Cronologicamente, este artigo apresenta os seguintes conteúdos: (a) conceitos de desenvolvimento sustentável e sustentabilidade, sustentabilidade em organizações e criação de valor sustentável; (b) cognição nas organizações, grau de complexidade cognitiva e mapas cognitivos; (c) procedimentos metodológicos; (d) resultados e análises; (e) considerações finais.

\section{Desenvolvimento Sustentável e Sustentabilidade}

O sistema capitalista, impulsionado pela Revolução Industrial a partir do século XVIII, beneficiou sociedades com melhores padrões de vida nunca observados anteriormente (Easterlin, 2000; Johnson, 2000). Sociedades puderam usufruir de melhorias em suas rendas, saúde, expectativa de vida, infraestrutura e saneamento urbano, alimentação, mobilidade, moradia, vestuário, entre outras. Transformações de conjuntura econômica, social e política estimularam o desenvolvimento de ciência e tecnologia que resultou em inovações nas áreas de produção, energia, química, medicina, comunicação e computação, construção civil, também entre outras (Gordon, 2000; Nobre, Walker, \& Harris, 2012). Essas transformações estimularam a criação de organizações industriais e corporações modernas que se beneficiaram de desenvolvimentos tecnológicos para a estruturação de seu capital e, portanto, de seus sistemas de produção, com a finalidade de maximização da produtividade, do consumo, e, por conseguinte, do lucro (Nobre, Tobias, \& Walker, 2009). Essa revolução também 
representou uma maior ruptura nas relações entre indivíduo e sociedade, e, especialmente, entre homem e natureza, em que se estabeleceram caminhos para um crescimento econômico predatório (Hawken, Lovins, \& Lovins, 1999; Vivien, 2011). A exploração e o consumo demasiados de recursos naturais sem assegurar a sua reprodução, associados a fatores como a globalização, o crescimento populacional (que se expandiu aproximadamente de 1 para 7 bilhões de pessoas nos últimos 200 anos), e a discrepância na distribuição da riqueza mundial, demonstram a real natureza de parte de um problema que permeia a sociedade (Ehrlich, 1968; Kandachar \& Halme, 2008; Prahalad, 2004; Prahalad \& Hart, 2002; Rogers, Jalal, \& Boyd, 2008). Diante desse panorama, destaca-se um movimento em busca de um novo caminho para um desenvolvimento sustentável.

O desenvolvimento sustentável é um conceito dinâmico, em constante evolução, que tem assumido diversas definições na literatura, sendo contingencial a contextos culturais, interdisciplinares, temporais e locais (Gladwin, Kennelly, \& Krause, 1995; Rogers et al., 2008). A sua formação como conceito, mais especificamente na economia, evoluiu através de reflexões de pesquisadores a partir da Revolução Industrial (Vivien, 2011); contudo, as suas premissas receberam maior atenção e difusão a partir da metade do século XX, especialmente através das conferências mundiais da Organização das Nações Unidas em Estocolmo (1972), Rio de Janeiro (1992 e 2012), Quioto (1997) e Joanesburgo (2002); de publicações do Clube de Roma, como The Limits to Growth (Meadows, Meadows, Randers, \& Behrens, 1972), e da Organização das Nações Unidas (ONU), especialmente da Comissão Mundial sobre Meio Ambiente e Desenvolvimento, como Nosso Futuro Comum, também conhecido por Relatório Brundtland (WCED, 1987). Em Nosso Futuro Comum (WCED, 1987), definiu-se que o desenvolvimento sustentável deve garantir o atendimento às necessidades do presente sem comprometer a capacidade das futuras gerações atenderem às suas necessidades. Nessa perspectiva, a sustentabilidade pode ser compreendida como um conjunto de valores e critérios, entre os quais se pode incluir a sustentabilidade social, cultural, ecológica, ambiental, territorial, econômica e política (Sachs, 2000), que, quando satisfeitos, atendem às necessidades do presente e do futuro, possuindo, portanto, um papel central nas reflexões em torno do desenvolvimento; e o desenvolvimento sustentável pode ser compreendido como caminhos ou conjuntos de estratégias planejadas para satisfazerem critérios da sustentabilidade, uma vez que o desenvolvimento pressupõe uma transformação progressiva e deliberada da sociedade, da economia e do ecossistema (Nobre et al., 2012; Richter, 1982), em que ecossistema compreende a coesão de uma comunidade de organismos e o seu ambiente (Beeby \& Brennan, 2007). Gladwin, Kennelly e Krause (1995) definem que o desenvolvimento sustentável envolve um processo para se alcançar o desenvolvimento humano de uma maneira inclusiva, interligada, igualitária, prudente e segura. Segundo a UNESCO (2005, p. 41), o objetivo final é "alcançar a coexistência pacífica entre os povos, reduzir o sofrimento, a fome e a pobreza, em um mundo onde as pessoas possam exercer dignamente seus direitos como seres humanos e cidadãos", em que se ressalta a necessidade de uma constante abordagem holística para compreender as complexidades nas inter-relações entre o meio ambiente e o homem.

\section{Sustentabilidade em organizações (SEO)}

A inserção do conceito da sustentabilidade nas organizações e, mais especificamente, no contexto de empresas, corporações e negócios, recebeu especial atenção quando John Elkington cunhou o termo Tripé da Sustentabilidade, originalmente Triple Bottom Line (TBL). O TBL propõe uma visão multidimensional que integra três dimensões correspondentes a valores e a resultados de uma organização medida em termos sociais, ambientais e econômicos (Elkington, 1994, 1997, 2004). A sustentabilidade na organização, desde então, passou a ser compreendida como a sua atenção de igual valor às questões social, ambiental e econômica. Nesse sentido, as empresas passam a desempenhar um papel central como agentes participantes no desenvolvimento sustentável ao criarem estratégias que contribuem para a concretização de objetivos e a satisfação de critérios da sustentabilidade.

Devido à disseminação dos conceitos propostos por Elkington, outros autores adotaram e estenderam a sua perspectiva para definirem organizações sustentáveis, estratégias de criação de valor 
sustentável nas organizações e nos indicadores de sustentabilidade empresarial. Ao definir uma organização inovadora sustentável, Barbieri, Vasconcelos, Andreassi e Vasconcelos (2010, p. 150) mencionam que "Não basta, para as empresas, apenas inovar constantemente, mas inovar considerando as três dimensões da sustentabilidade". Hart e Milstein (2003) definem uma empresa sustentável como aquela que contribui para o desenvolvimento sustentável à medida que gera benefícios econômicos, sociais e ambientais, referindo-se ao TBL. Nessas empresas, a sustentabilidade pode ser compreendida como fonte propulsora de inovações (Nidumolu, Prahalad, \& Rangaswami, 2009; Nobre et al., 2012). Tratando-se da formulação de estratégias que contribuem para a busca da sustentabilidade nas organizações, Hart (2005) e, Hart e Milstein (2003) propuseram um modelo de criação de valor sustentável. Esse modelo, quando sistemicamente adotado, pode contribuir para a melhoria do desempenho econômico, social e ambiental da empresa. Por final, considerando-se a necessidade de avaliação, mensuração e controle das dimensões da sustentabilidade empresarial, e da busca por maior transparência nos negócios, alguns indicadores de sustentabilidade foram propostos especialmente para empresas participantes no mercado de capitais (BM\&FBOVESPA, n.d.; Marcondes \& Bacarji, 2010).

Outro fenômeno que também contribui para o desenvolvimento sustentável é o envolvimento das empresas com relação aos conceitos e às práticas de Responsabilidade Social Empresarial (RSE) (Aguinis \& Glavas, 2012; Barbieri \& Cajazeira, 2012; Dahlsrud, 2008; Garriga \& Melé, 2004). Precedente ao conceito de sustentabilidade nas organizações, as publicações relacionadas à RSE tiveram início na primeira metade do século XX (Aguinis \& Glavas, 2012), e a relevância desse tema foi enfatizada pela ONU em 2000 com a criação do Pacto Global, que propõe 10 princípios universais para nortear empresas signatárias (United Nations Global Compact [UNGC], 2011). Embora possua conceitos relacionados à sustentabilidade, a RSE não será tema deste artigo, que tem o seu foco sobre o modelo de Criação de Valor Sustentável (Hart \& Milstein, 2003) e o Índice de Sustentabilidade Empresarial (BM\&FBOVESPA, n.d.).

\section{Criação de valor sustentável (CVS): foco em estratégias}

A Criação de Valor Sustentável (CVS) (Hart, 2005; Hart \& Milstein, 2003) teve sua origem a partir de conceitos da Visão da Empresa Baseada em Recursos Naturais (VBRN) (Hart, 1995; Hart \& Dowell, 2011), em que a VBRN está fundamentada em publicações clássicas sobre Visão da Empresa Baseada em Recursos (VBR) e capacidades dinâmicas (Barney, 1991, 1996; Teece, Pisano, \& Shuen, 1997). Adicionalmente, conceitos da VBRN e, portanto, da CVS, também foram estimulados em meio à crescente discussão de temas relacionados ao desenvolvimento sustentável, incluindo sustentabilidade ambiental (Goodland, 1995), base da pirâmide (Prahalad, 2004; Prahalad \& Hart, 2002), inovação disruptiva e tecnologia limpa (Hart, 1997; Hart \& Christensen, 2002), entre outros. Em resumo, a VBRN representa uma tentativa de extensão da VBR; primeiro, ao incluir os recursos naturais como elementos essenciais para criação de vantagem competitiva das empresas; e, segundo, ao explicar que a omissão da relação entre a organização e o seu meio ambiente (natural) pode representar uma ameaça à sobrevivência da empresa que procura sustentar a sua vantagem competitiva. Adicionalmente, Hart (1995) explica que a estratégia e a vantagem competitiva das empresas estarão cada vez mais fundamentadas em capacidades que facilitem e proporcionem atividades econômicas ambientalmente sustentáveis. Com base nessa premissa, Hart (1995) propõe três estratégias principais denominadas de Combate à Poluição, Gerenciamento do Produto e Desenvolvimento Sustentável, as quais representam distintas e complementares forças ambientais e fontes de vantagem competitiva. A partir da evolução de outros conceitos relacionados à sustentabilidade, nas décadas de 1990 e 2000, Hart e Dowell (2011) adicionaram outras estratégias à VBRN, destacando-se Tecnologia Limpa (Hart, 1997) e Base da Pirâmide (Prahalad, 2004), sendo que esta última é uma proposta relacionada à erradicação da pobreza no mundo, e, portanto, foi inserida no contexto da estratégia do Desenvolvimento Sustentável introduzida na primeira publicação sobre a VBRN, em Hart, 1995. Portanto, por estar fundamentada nos conceitos da VBRN, a Criação de Valor Sustentável (CVS) (Hart, 2005; Hart \& Milstein, 2003) propõe um conjunto de quatro estratégias principais que, quando simultaneamente adotadas e praticadas, podem contribuir significativamente para a melhoria do desempenho econômico, social e ambiental das empresas. 
Para fins de operacionalização, Hart e Milstein (2003) propuseram um modelo de duas dimensões que inclui as quatro estratégias principais de Criação de Valor Sustentável (CVS) em um plano de quatro quadrantes, sendo que cada uma das estratégias está associada a um quadrante, como ilustrado na Figura 1. O eixo horizontal destaca os ambientes interno e externo da organização, e o eixo vertical distingue focos de curto (hoje ou presente) e longo prazo (amanhã ou futuro) da organização. A combinação dessas duas dimensões permite identificar que as estratégias de Combate à Poluição (CP) e Gerenciamento do Produto (GP) estão classificadas como capacidades internas com foco no presente da organização, e as estratégias de Tecnologia Limpa (TL) e Base da Pirâmide (BP) estão classificadas como capacidades externas com foco no futuro da organização. Hart e Milstein (2003) enfatizam que a adoção dessas quatro estratégias em conjunto proporcionará maiores benefícios e vantagens para a empresa, a comunidade e o meio ambiente. Em sua pesquisa empírica, Kurapatskie e Darnall (2013) classificaram empresas que adotam suas estratégias com foco nos dois quadrantes de baixo do modelo de CVS como Empresas Sustentáveis de Baixa Ordem (ESBO), e aquelas com foco nos dois quadrantes de cima do modelo de CVS como Empresas Sustentáveis de Alta Ordem (ESAO). Esses autores demonstraram que essas duas classes de empresas têm as suas estratégias associadas a um desempenho econômico superior, mas que os benefícios financeiros de ESAO são, em média, superiores àqueles de ESBO.

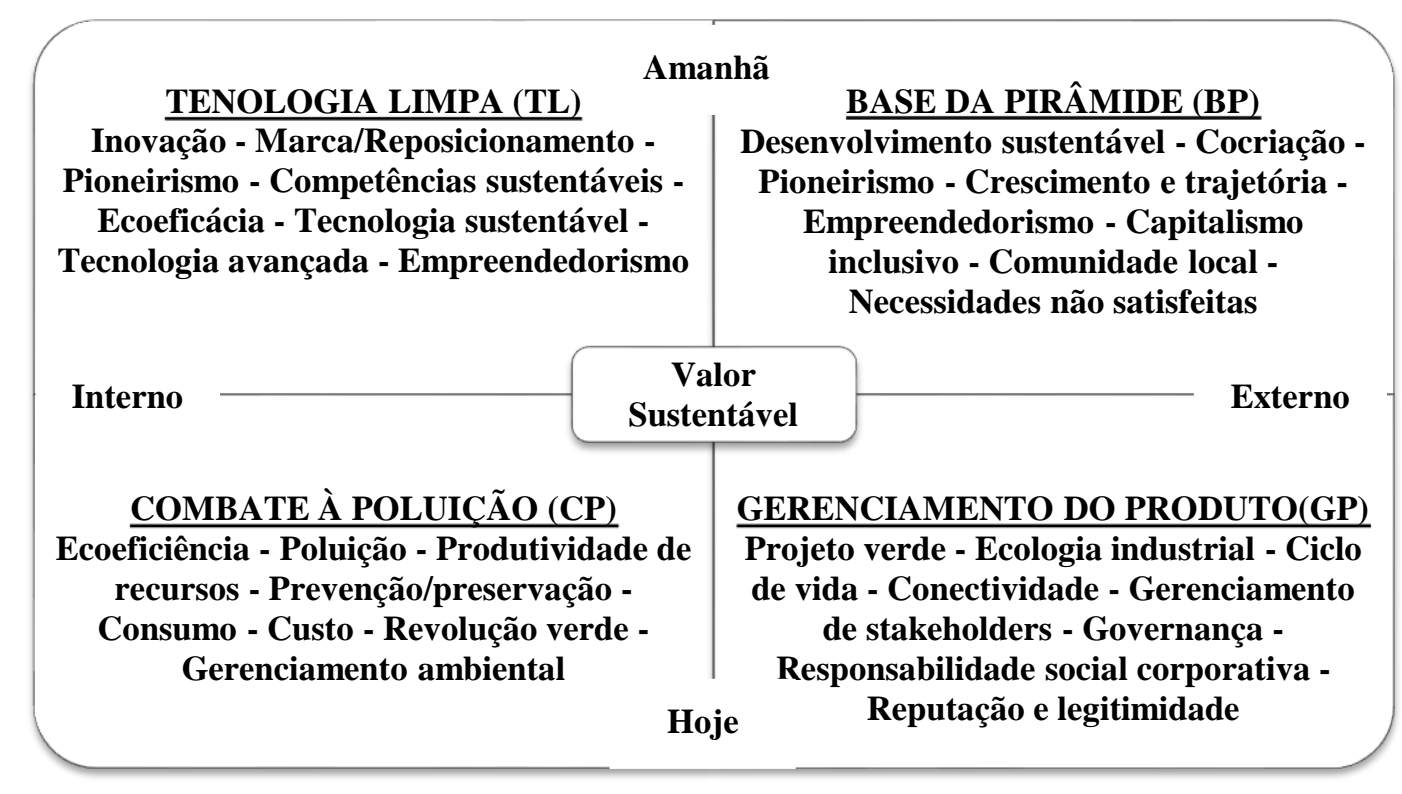

Figura 1. Modelo de Criação de Valor Sustentável (CVS).

Fonte: Adaptado de Hart, S. L. (2005). Capitalism at the crossroads: the unlimited business opportunities in solving the world's most difficult problems (p. 60). Upper Saddle River, NJ: Wharton School Publishing, e Hart, S. L., \& Milstein, M. B. (2003). Creating sustainable value (p. 88). Academy of Management Executive, 17(2), 56-67.

Esta pesquisa adota esse modelo de CVS para orientar a elaboração e a análise dos mapas cognitivos que serão construídos a partir dos relatórios de sustentabilidade das empresas estudadas.

\section{Índice de sustentabilidade empresarial (ISE): foco em indicadores de desempenho}

A BM\&FBOVESPA criou e mantém uma série de índices para realizar o acompanhamento do desempenho de empresas que buscam as melhores práticas de responsabilidade social e sustentabilidade. Esses índices servem também de guia para investidores interessados no retorno econômico associado a critérios sociais e ambientais (BM\&FBOVESPA, n.d.). Entre tais índices, está incluído o Índice de Sustentabilidade Empresarial (ISE) da BM\&FBOVESPA, que foi criado em 2005 para proporcionar um ambiente de investimento que estivesse alinhado às demandas do desenvolvimento sustentável e ao estímulo da ética empresarial. O ISE possui sete dimensões principais, denominadas Geral, Natureza do Produto, Governança Corporativa, Econômico- 
Financeira, Social, Ambiental e Mudanças Climáticas, que são norteadoras de processos de avaliação de desempenho de sustentabilidade das empresas (BM\&FBOVESPA, 2011; Centro de Estudos em Sustentabilidade, 2012a, 2012b)

\section{Cognição nas Organizações}

Cognição abrange processos e representações nas organizações (Nobre et al., 2010, 2011). Por um lado, quando associada a processos, a cognição exerce a função de mediadora de efeitos de eventos externos ou estímulos sobre as decisões, os comportamentos e as ações de indivíduos, grupos e organizações, em resposta às suas experiências. Por outro lado, quando associada a representações, a cognição se torna sinônimo de imagens mentais, modelos de conhecimento e mapas cognitivos elaborados a partir da interpretação, da experiência e da aprendizagem de indivíduos, grupos e organizações.

\section{Grau de complexidade cognitiva (GCC)}

Neste trabalho, o conceito de Grau de Complexidade Cognitiva (GCC) é compreendido como uma medida ou representação simbólica do nível de elaboração com que os participantes nas organizações percebem e interpretam seus ambientes para formulação e escolha de estratégias (Calori, Johnson, \& Sarnin, 1994; Nobre et al., 2009, 2010, 2011; Nobre \& Walker, 2011a, 2011b; Porac \& Thomas, 2002). Operacionalmente, um GCC é representado por uma medida relacionada à diferenciação e à integração de um mapa cognitivo (Calori et al., 1994; Walsh, 1995), em que a Diferenciação (D) equivale ao número de construtos (ou categorias), e a Integração (I), ao número de links (ou relações entre as categorias e subcategorias) presentes em um mapa cognitivo.

\section{Mapas cognitivos}

Conceitualmente, este artigo adota a definição de que mapas cognitivos são representações mentais resultantes de processos cognitivos como sentido, percepção, atenção, identificação e formação de conceitos, categorização, memória, linguagem, decisão, aprendizagem e resolução de problemas (Nobre et al., 2010, 2011; Nobre \& Walker, 2011a, 2011b). Mapas cognitivos podem ser simbolica e explicitamente representados na forma de conceitos ou construtos, padrões de categorias e relações (links) causais (Eden, 1992, 2004; Fiol \& Huff, 1992). Operacionalmente, os mapas cognitivos, e, portanto, os seus construtos e links (relações) foram elaborados neste trabalho a partir da análise de conteúdo dos relatórios de sustentabilidade das organizações estudadas; em que, a medida do Grau de Complexidade Cognitiva (GCC) de cada organização é uma função da diferenciação e da integração do seu respectivo mapa cognitivo. Todos os mapas cognitivos nesta pesquisa foram construídos com o auxílio do software Inspiration.

\section{GCC vs. SEO: uma possível relação}

Considerando-se os conceitos apresentados, esta pesquisa contribui ao investigar a existência de uma possível relação entre Grau de Complexidade Cognitiva (GCC) e Sustentabilidade em Organizações (SEO) de empresas que participam no Índice de Sustentabilidade Empresarial (ISE) da Bolsa de Valores de São Paulo (BM\&FBOVESPA).

\section{Procedimentos Metodológicos}

Como metodologia de pesquisa, adotou-se uma abordagem qualitativa, conduzida por meio de estudo de casos múltiplos (Eisenhardt, 1989; Yin, 2005), com perspectiva temporal transversal. A 
amostra intencional foi constituída por seis empresas que disponibilizaram por completo seus relatórios de sustentabilidade e questionários do ISE, sendo elas: AES Tietê, Banco do Brasil, BICBanco, Coelce, Eletropaulo e Natura. Análises de conteúdo foram realizadas sobre o modelo de Criação de Valor Sustentável (CVS) (Hart, 1995, 1997, 2005; Hart \& Dowell, 2011; Hart \& Milstein, 2003), os relatórios de sustentabilidade, o Índice de Sustentabilidade Empresarial (ISE) (Centro de Estudos em Sustentabilidade, 2012b) e os questionários do ISE das empresas. As análises de conteúdo seguiram as etapas de pré-análise, exploração do material, tratamento dos resultados, inferência e interpretação (Bardin, 2009; Mozzato \& Grzybovski, 2011). Resultados das análises de conteúdo proporcionaram elementos para determinação de medidas do Grau de Complexidade Cognitiva (GCC) e da Sustentabilidade em Organizações (SEO) das empresas estudadas. Portanto, cada empresa foi associada a um par de valores (GCC, SEO).

\section{Medida de grau de complexidade cognitiva (GCC)}

A partir de análises de conteúdo do modelo de Criação de Valor Sustentável (CVS) e dos relatórios de sustentabilidade das empresas, identificaram-se categorias e subcategorias que permitiram a elaboração de mapas cognitivos (Bastos, 2002; Eden, 1992, 2004; Fiol \& Huff, 1992).

Em uma primeira etapa, elaborou-se um mapa cognitivo representando simbolicamente o modelo de CVS, como ilustrado na Figura 2. O mapa mostra 4 estratégias principais, em que cada estratégia está relacionada a um conjunto de 8 construtos, totalizando, portanto, 32 construtos. Esse mapa foi denominado de protótipo de CVS por servir de referência para a análise do Grau de Complexidade Cognitiva (GCC) de cada uma das empresas.

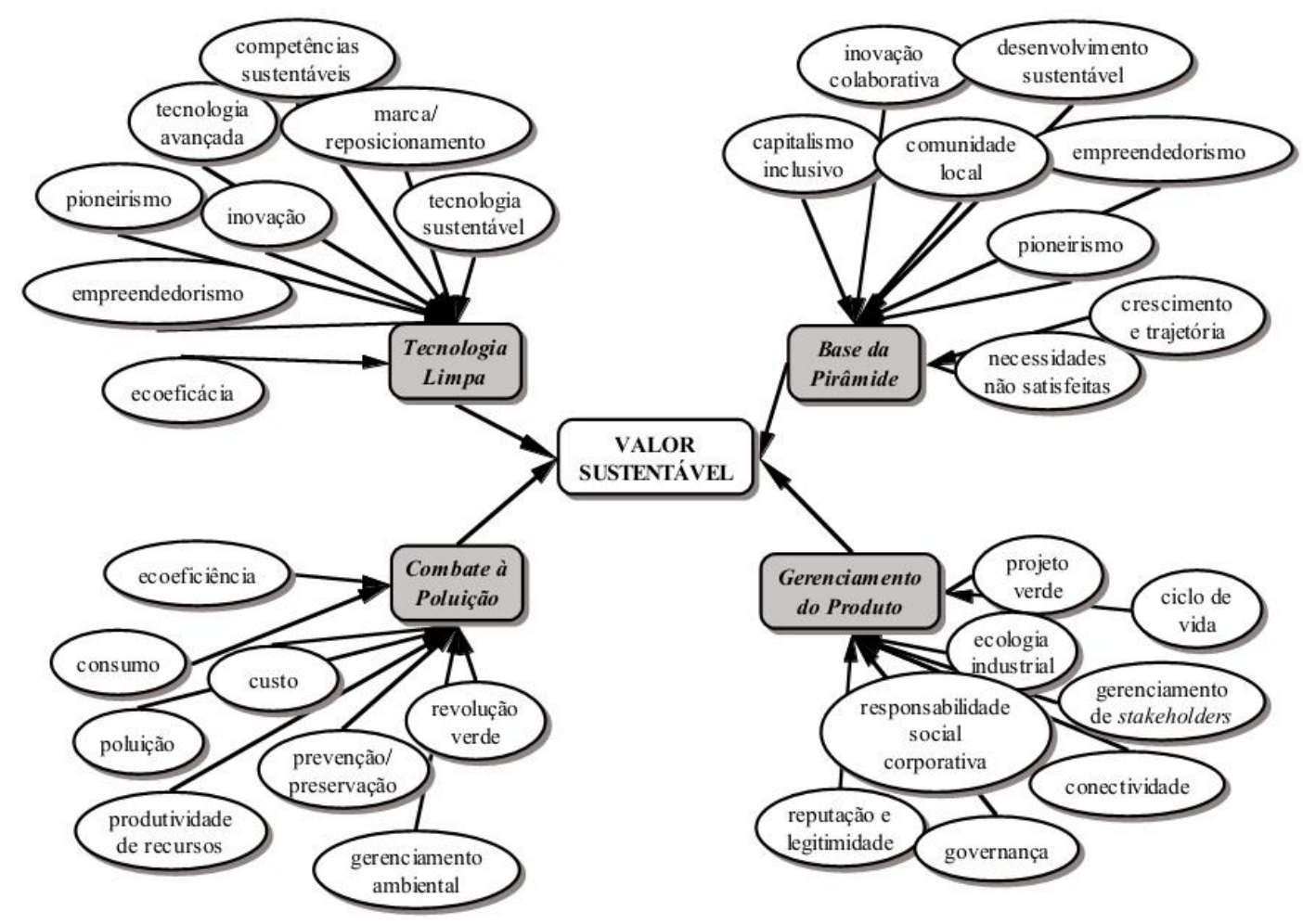

Figura 2. Mapa Protótipo de CVS.

Em seguida, elaborou-se um conjunto de seis mapas cognitivos a partir das análises de conteúdo dos relatórios de sustentabilidade das empresas disponibilizados nos seus respectivos sítios. Esses seis mapas cognitivos foram elaborados adotando o protótipo de CVS como referencial e, por isso, foram denominados de mapa CVS da empresa $\boldsymbol{x}$. A sua estrutura foi constituída por uma camada principal contendo as 4 estratégias do modelo de CVS; uma segunda camada foi formada contendo construtos 
identificados através da análise de conteúdo e relacionados às 4 estratégias da camada principal, em que, de acordo com o protótipo de CVS, um máximo de 8 construtos poderiam estar associados a cada uma das 4 estratégias da camada principal; e uma terceira camada contendo subcategorias ou links que representam práticas ou ações relacionadas aos construtos da segunda camada. Cada empresa foi então associada a um mapa cognitivo e, a partir da diferenciação e da integração de cada mapa, calculou-se um valor de Grau de Complexidade Cognitiva (GCC) correspondente a cada empresa.

O valor absoluto do Grau de Complexidade Cognitiva (GCC) de cada uma das seis empresas foi calculado a partir da seguinte medida:

$$
M G C C_{x=(1, \ldots, 6)}=D_{x=(1, \ldots, 6)}+I_{x=(1, \ldots, 6)}
$$

$M G C C_{x=(1, \ldots, 6)}$ - Medida absoluta do GCC da empresa $\mathrm{x}$, em que x representa uma variável que assume valores inteiros e reais no intervalo discreto $[0,6]$.

$D_{x=(1, \ldots, 6)}-$ Diferenciação (número de construtos) encontrada no mapa cognitivo da empresa $\mathrm{x}$.

$I_{x=(1, \ldots, 6)}$ - Integração (número de links) encontrada no mapa cognitivo da empresa x.

- Nota: Esta definição de MGCC foi derivada de conceitos relacionados à Teoria Geral de Sistemas (Bertalanffy, 1968; Boulding, 1956). Alguns trabalhos que abordam a complexidade como função da diferenciação e da integração incluem Boulding (1956); Bunge e Ardila (1987); Hall e Fagen (1956); Nobre, Tobias e Walket (2010); e Simon (1996). Nesses trabalhos, a complexidade de um sistema é função da sua estrutura e, portanto, do número de elementos e interconexões contidas no sistema, em que elemento é sinônimo de construto e interconexão é sinônimo de integração (link) (Nobre et al., 2010).

E o seu valor normalizado correspondente foi calculado por:

$$
M G C C_{x=(1, \ldots, 6)}^{\prime}=\frac{\left[D_{x=(1, \ldots, 6)}+I_{x=(1, \ldots, 6)}\right]}{M G C C_{M}} * 100
$$

- $M G C C^{\prime}{ }_{x=(1, \ldots, 6)}$ - Medida normalizada do GCC da empresa x.

$M G C C_{M}$ - Medida absoluta do maior MGCC das empresas, em que $\mathrm{MGCC}_{\mathrm{M}}$ representa o maior entre os valores de $\mathrm{MGCC}_{\mathrm{x}=(1, \ldots, 6)}$, ou seja, $\mathrm{MGCC}_{\mathrm{M}}=\max \left[\mathrm{MGCC}_{\mathrm{x}=(1, \ldots, 6)]}\right.$.

\section{Medida de sustentabilidade em organizações (SEO)}

Análises de conteúdo do Índice de Sustentabilidade Empresarial (ISE) permitiram a identificação de dimensões, critérios e indicadores, assim como os seus respectivos pesos, que foram utilizados como referência no cálculo da Sustentabilidade em Organizações (SEO) de cada uma das empresas. Verificou-se que o ISE é composto de 7 dimensões, 29 critérios e 73 indicadores (Centro de Estudos em Sustentabilidade, 2012b), sendo que indicadores são subclasses pertencentes aos critérios, e critérios são subclasses pertencentes às dimensões. Na metodologia do ISE, os pesos são definidos, principalmente, pela relevância do tema no contexto atual da gestão empresarial e das demandas da sociedade. Cada dimensão possui peso 100 que, multiplicado por 7 dimensões, totaliza 700. Cada critério, por sua vez, possui peso específico que varia de uma dimensão para outra, sendo que a soma dos critérios dentro de cada dimensão é igual a 100. Contudo, a metodologia do ISE não publica os pesos dos indicadores. Por conseguinte, convencionou-se, nesta pesquisa, atribuir um peso de igual valor para cada um dos indicadores relacionados a um mesmo critério, no qual a soma dos indicadores pertencentes a um critério é igual ao peso do próprio critério. Como exemplo, o critério Compromissos contido na dimensão Geral possui peso 15 e está associado a dois indicadores, IG-1 e IG-2, cujos pesos estão definidos por 7,5. A Tabela 1 mostra as dimensões, os critérios e os indicadores do ISE. 
Tabela 1

Dimensões, Critérios e Indicadores do ISE

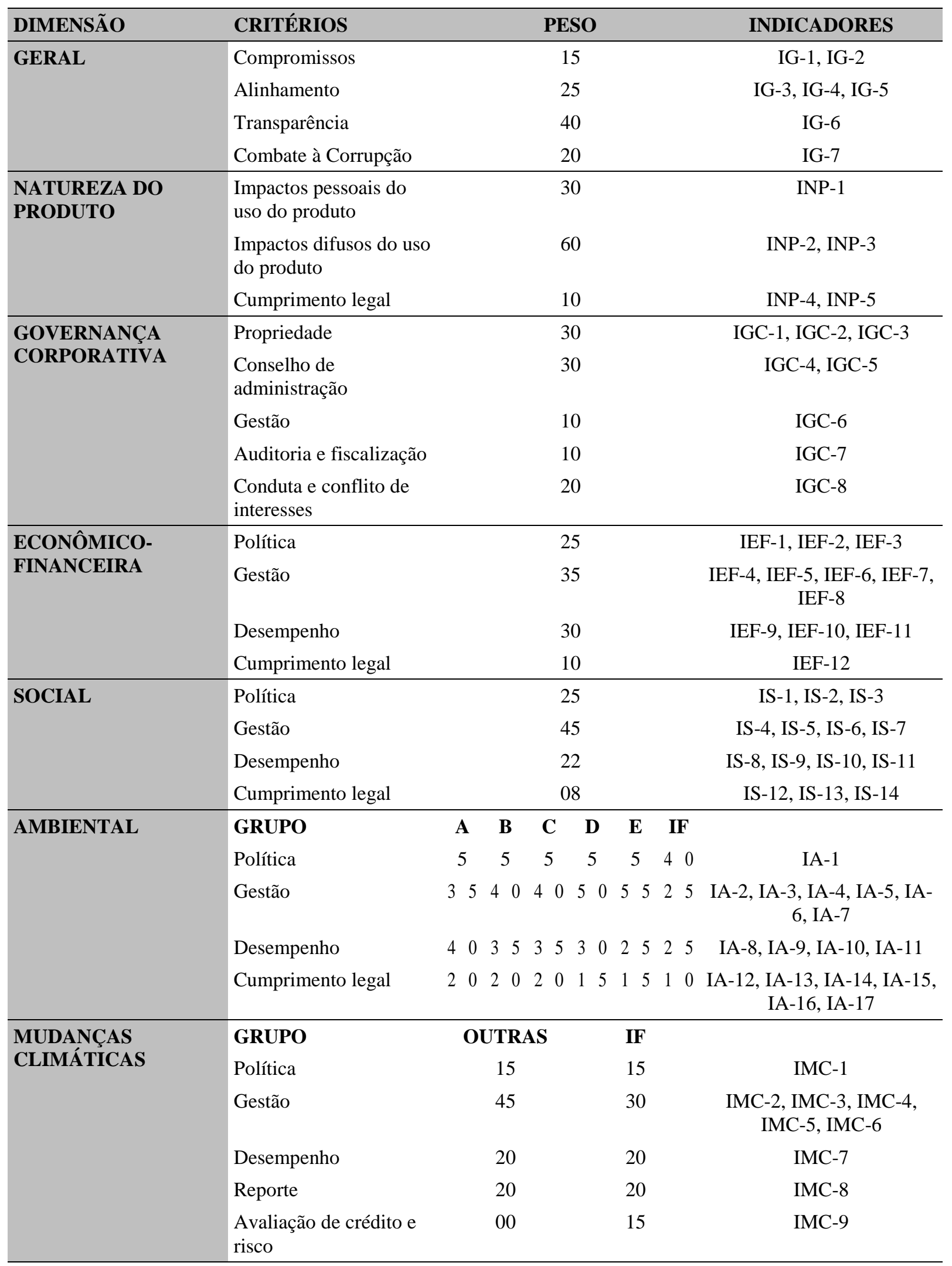


A partir dos questionários do ISE de cada empresa, calculou-se então a Sustentabilidade em Organizações (SEO). O valor normalizado da Sustentabilidade em Organizações (SEO) de cada uma das seis empresas foi calculado a partir da seguinte medida:

$$
\operatorname{MSEO}_{x=(1, \ldots, 6)} \frac{\sum_{n=1}^{7} D I S E_{x=(1, \ldots, 6)}^{n}}{700} * 100
$$

. MSEO $_{x=(1, \ldots, 6)}-$ Medida normalizada da SEO da empresa x.

. $D I S E_{x=(1, \ldots, 6)}-$ Medida das 7 dimensões do questionário do ISE da empresa $\mathrm{x}$.

\section{Desenho da pesquisa}

A Figura 3 ilustra o problema de pesquisa que compreende o questionamento da relação entre GCC e SEO, sendo simbolicamente representada pela seta pontilhada. A Figura 3 também mostra as quatro estratégias principais do modelo de CVS e as sete dimensões do ISE.

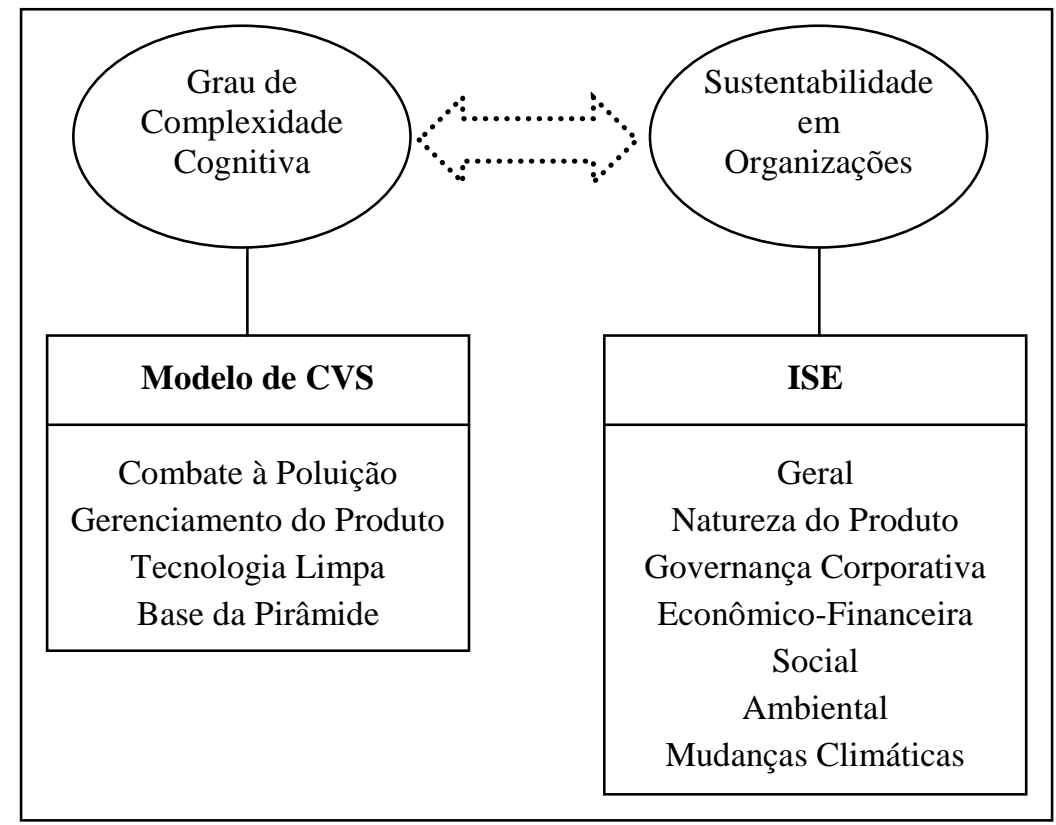

Figura 3. Ilustração do Problema de Pesquisa.

\section{Resultados e Análises}

As empresas que compõem o estudo de casos múltiplos são: AES Tietê, Banco do Brasil, BIC Banco, Coelce, Eletropaulo e Natura. A presença destas na carteira do ISE demonstra a participação e o envolvimento delas com a sustentabilidade. A Tabela 2 apresenta o histórico da participação dessas empresas no ISE desde o seu surgimento, em 2005. O ISE pode possuir até 40 empresas. Esse período de existência incluiu a participação de 64 delas. Algumas participam no índice em determinado período e, quando não cumprirem os critérios em determinado ano, são excluídas em seguida, e outras empresas passam a participar no índice. A Tabela 2 mostra que as seis empresas selecionadas já eram integrantes do índice no ano anterior a 2012, sendo que três delas - Banco do Brasil, Eletropaulo e Natura - participam no ISE desde a criação deste, em 2005. 
Tabela 2

Histórico de Participação das Empresas Estudadas no ISE, de 2005 a 2012

\begin{tabular}{lccccccc}
\hline & \multicolumn{1}{c}{ ÍNDICE DE SUSTENTABILIDADE EMPRESARIAL - ISE } & & \\
\hline DATA DA CARTEIRA & $01 / 12 / 05$ & $01 / 12 / 06$ & $03 / 12 / 07$ & $01 / 12 / 08$ & $01 / 12 / 09$ & $03 / 01 / 11$ & $02 / 01 / 12$ \\
AES TIETE & & & $\mathrm{x}$ & $\mathrm{x}$ & $\mathrm{x}$ & $\mathrm{x}$ & $\mathrm{x}$ \\
BIC BANCO & & & & $\mathrm{x}$ & $\mathrm{x}$ \\
BANCO DO BRASIL & $\mathrm{x}$ & $\mathrm{x}$ & $\mathrm{x}$ & $\mathrm{x}$ & $\mathrm{x}$ & $\mathrm{x}$ & $\mathrm{x}$ \\
COELCE & & $\mathrm{x}$ & $\mathrm{x}$ & $\mathrm{x}$ & $\mathrm{x}$ & $\mathrm{x}$ & $\mathrm{x}$ \\
ELETROPAULO & $\mathrm{x}$ & $\mathrm{x}$ & $\mathrm{x}$ & $\mathrm{x}$ & $\mathrm{x}$ & $\mathrm{x}$ & $\mathrm{x}$ \\
NATURA & $\mathrm{x}$ & $\mathrm{x}$ & $\mathrm{x}$ & $\mathrm{x}$ & $\mathrm{x}$ & $\mathrm{x}$ & $\mathrm{x}$ \\
\hline
\end{tabular}

TOTAL DE EMPRESAS QUE PASSARAM PELO ISE DE 2005 A 2012: 64 EMPRESAS

Nota. Fonte: BM\&FBOVESPA (2013). Carteiras Índice de Sustentabilidade Empresarial - ISE (Abertura). Recuperado de http://www.bmfbovespa.com.br/Indices/download/Evolutivo-das-carteiras-ISE.pdf

\section{Análise dos casos}

Os resultados de análises sobre os mapas CVS e questionários do ISE associados a cada empresa estão mostrados nas Tabelas 3 e 4 respectivamente.

Tabela 3

Dados Associados à CVS das Empresas Estudadas

CONSTRUTOS LINKS

\begin{tabular}{|c|c|c|c|c|c|c|c|c|c|c|c|c|c|}
\hline ESTRATÉGIA & 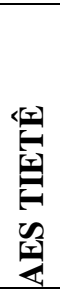 & 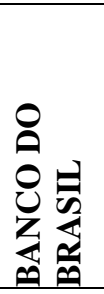 & 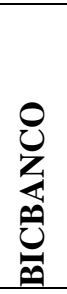 & $\begin{array}{l}\text { 된 } \\
\text { 종 } \\
0\end{array}$ & 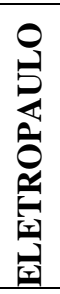 & 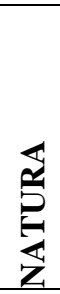 & 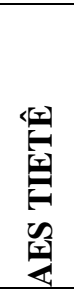 & 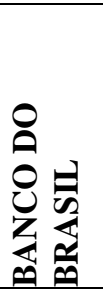 & 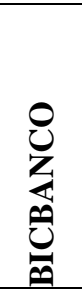 & $\begin{array}{l}\text { 된 } \\
\text { T્રે } \\
0 \\
\end{array}$ & 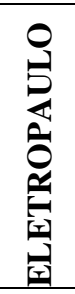 & 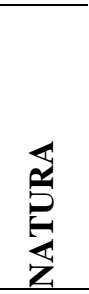 & 夜 \\
\hline $\begin{array}{l}\text { Combate à } \\
\text { Poluição }\end{array}$ & 3 & 5 & 4 & 4 & 4 & 4 & 9 & 15 & 10 & 12 & 14 & 10 & 94 \\
\hline $\begin{array}{l}\text { Gerenciamento do } \\
\text { Produto }\end{array}$ & 5 & 6 & 6 & 6 & 6 & 6 & 30 & 36 & 26 & 21 & 36 & 29 & 213 \\
\hline Base da Pirâmide & 2 & 5 & 2 & 2 & 2 & 4 & 2 & 5 & 5 & 8 & 10 & 6 & 53 \\
\hline Tecnologia Limpa & 5 & 5 & 4 & 4 & 4 & 7 & 10 & 5 & 5 & 9 & 6 & 11 & 75 \\
\hline TOTAL & 15 & 21 & 16 & 16 & 16 & 21 & 51 & 75 & 46 & 50 & 66 & 56 & ---- \\
\hline TOTAL (\%) & 47 & 66 & 50 & 50 & 50 & 66 & ---- & ---- & ---- & ---- & ---- & ---- & ---- \\
\hline
\end{tabular}

Valores das medidas absoluta $\left(M G C C_{x}\right)$ e normalizada $\left(M G C C_{x}{ }_{x}\right)$ do GCC das empresas estudadas

\begin{tabular}{|c|c|c|c|c|c|c|}
\hline & 里罢 & 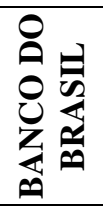 & $\begin{array}{l}0 \\
Z \\
z \\
0 \\
0 \\
0\end{array}$ & 秥 & 芴 & 兑 \\
\hline$M G C C_{x}$ & 66 & 96 & 62 & 66 & 82 & 77 \\
\hline$M G C C_{x}^{\prime}$ & 68,8 & 100 & 64,6 & 68,8 & 85,4 & 80,2 \\
\hline
\end{tabular}


Tabela 4

Dados Associados aos Questionários do ISE das Empresas Estudadas

\begin{tabular}{|c|c|c|c|c|c|c|c|}
\hline \multirow[b]{2}{*}{ DIMENSÃO } & \multicolumn{6}{|c|}{ NÍVEL DE ATENDIMENTO } & \multirow[b]{2}{*}{ TOTAL } \\
\hline & $\begin{array}{c}\text { AES } \\
\text { TIETÊ }\end{array}$ & $\begin{array}{l}\text { BANCO } \\
\text { DO } \\
\text { BRASIL }\end{array}$ & $\begin{array}{c}\text { BIC } \\
\text { BANCO }\end{array}$ & COELCE & ELETROPAULO & NATURA & \\
\hline Geral & 83,6 & 89 & 72,6 & 78,4 & 83,5 & 86,3 & 493,4 \\
\hline $\begin{array}{l}\text { Natureza do } \\
\text { Produto }\end{array}$ & 99,5 & 58,3 & 75,5 & 99 & 99 & 84,8 & 516,1 \\
\hline $\begin{array}{l}\text { Governança } \\
\text { Corporativa }\end{array}$ & 67,7 & 83,6 & 68,7 & 62,5 & 75,1 & 82,8 & 440,4 \\
\hline $\begin{array}{l}\text { Econômico- } \\
\text { Financeira }\end{array}$ & 46,5 & 49 & 58,9 & 34,4 & 45,6 & 55,8 & 290,2 \\
\hline Social & 56,9 & 69,6 & 55,7 & 71,5 & 60,5 & 55,3 & 369,5 \\
\hline Ambiental & 55,1 & 62,7 & 54,2 & 51,7 & 54,9 & 49 & 327,6 \\
\hline $\begin{array}{l}\text { Mudanças } \\
\text { Climáticas }\end{array}$ & 45,8 & 54,8 & 28,3 & 45,9 & 54,8 & 58,4 & 288 \\
\hline$\sum D I S E_{x}$ & 455,1 & 467 & 413,9 & 443,4 & 473,4 & 472,4 & ---- \\
\hline $\operatorname{MSEO}_{x}$ & 65,0 & 66,7 & 59,1 & 63,3 & 67,6 & 67,5 & ----- \\
\hline
\end{tabular}

Em uma análise sobre a Tabela 3, verificou-se que os casos estudados adotam e priorizam estratégias similares, apresentam uma quantidade de construtos similar e diferenciam-se em relação aos links, o que pode ser decorrente dos seus diferentes segmentos de atuação. Verificou-se que os casos estudados concentram maior atenção em práticas e ações relacionadas à estratégia de Gerenciamento de Produto, na qual a soma dos construtos e links totaliza 213. Em segundo lugar, está o Combate à Poluição, que somou 94; em terceiro, a Tecnologia Limpa, a qual somou 75; e, em quarto, a Base da Pirâmide, que somou 53. Hart (2005), e Hart e Milstein (2003) explicam que as empresas têm maior facilidade de trabalhar no campo inferior do modelo de Criação de Valor Sustentável (CVS), que comporta as duas estratégias principais do presente, mostradas na Figura 1. Esses autores afirmam que boa parte das empresas adotará e desenvolverá atividades relacionadas às estratégias de Gerenciamento do Produto e Combate à Poluição. Com base nos resultados apresentados na Tabela 3, verifica-se que esta pesquisa corrobora com as observações desses autores.

Em outra análise sobre a Tabela 4, verificou-se que a dimensão com melhor desempenho para o conjunto de casos é a Natureza do Produto, em que a soma dos valores totaliza 516,1; e que a dimensão com menor desempenho para o conjunto de casos se refere a Mudanças Climáticas, que totaliza 288. A dimensão Econômico-Financeira obteve a segunda menor pontuação para o conjunto de casos, totalizando 290,2, despertando curiosidade, uma vez que se trata de um índice referente às empresas integrantes da Bolsa de Valores de São Paulo (BM\&FBOVESPA). Contudo, não se pode concluir a partir desse resultado que as empresas tenham problemas referentes à sustentabilidade econômico-financeira, pois, em uma análise sobre os questionários do ISE respondidos por elas, verificou-se que, para essa dimensão, as empresas não apresentam comprovação de determinados critérios ou não fazem um devido controle e acompanhamento, o que determina uma menor pontuação. Adicionalmente, com base nos resultados empíricos obtidos por Kurapatskie and Darnall (2013), espera-se que o desempenho econômico-financeiro das empresas estudadas aumente à medida que essas aumentarem seus esforços na adoção de estratégias dos dois quadrantes superiores do modelo de Criação de Valor Sustentável (CVS). 
Analisando-se as Tabelas 3 e 4, verificou-se a existência de uma relação entre os valores de $M G C C^{\prime}{ }_{x}$ e $M S E O_{x}$. A Figura 4 ilustra esta relação que se caracteriza com tendência positiva, em que, à medida que o Grau de Complexidade Cognitiva (GCC) aumenta, verifica-se um aumento da Sustentabilidade em Organizações (SEO).

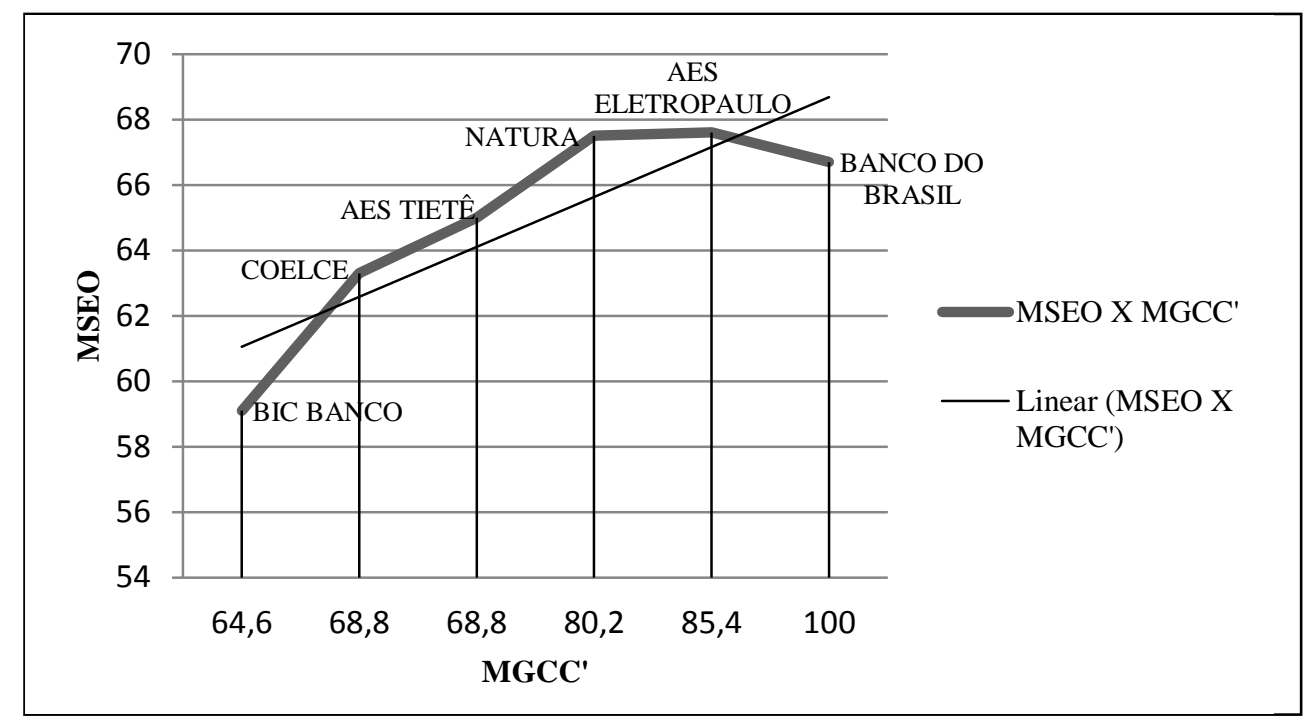

Figura 4. MSEO vs. MGCC'.

Analisando-se a Figura 4, observa-se que a Natura e a Eletropaulo apresentam valores de $M S E O$ muito similares e divergem pouco em relação aos valores de $M G C C^{\prime}$. Fato relevante é sobre o Banco do Brasil que, apesar de possuir o maior valor de $M G C C$ ' entre os casos estudados, apresenta um valor de $M S E O$ próximo de, embora um pouco menor que, os valores da Natura e da Eletropaulo, o que demanda maiores investigações para explicar a tendência verificada.

\section{Considerações Finais}

A sustentabilidade é um conceito interdisciplinar em constante evolução, sendo contingencial a contextos culturais, temporais e locais. Quando inserida nas organizações, a sustentabilidade demanda de seus participantes uma complexidade cognitiva mais elevada para interpretação das suas dimensões sistêmicas e dos seus múltiplos critérios associados à economia, à sociedade e ao meio ambiente.

Este artigo contribuiu ao estudar a relação entre Grau de Complexidade Cognitiva (GCC) e Sustentabilidade em Organizações (SEO) de empresas listadas no Índice de Sustentabilidade Empresarial (ISE) da Bolsa de Valores de São Paulo (BM\&FBOVESPA). Resultados de análises indicaram a existência de uma relação com tendência positiva entre GCC e SEO. Adicionalmente, este trabalho contribuiu para o campo dos estudos organizacionais e de estratégia ao elaborar representações da complexidade cognitiva, por meio de mapas cognitivos, para analisar de uma maneira alternativa a questão da sustentabilidade em organizações.

A pesquisa adotou uma abordagem qualitativa, conduzida por meio de estudo de casos múltiplos, com perspectiva temporal transversal. A amostra intencional foi constituída por seis empresas que disponibilizaram por completo seus relatórios de sustentabilidade e questionários do ISE. Foram elas: AES Tietê, Banco do Brasil, BICBanco, Coelce, Eletropaulo e Natura. A partir de análises de conteúdo dos relatórios de sustentabilidade destas, identificaram-se categorias e subcategorias que permitiram a elaboração de mapas cognitivos, tendo sido associado um para cada empresa e, em função da diferenciação e da integração de cada mapa, calculou-se um valor de Grau de 
Complexidade Cognitiva (GCC) para cada uma delas. Análises de conteúdo sobre os questionários do ISE permitiram a identificação de dimensões, critérios e indicadores, assim como os seus respectivos pesos, que foram utilizados no cálculo de um valor de Sustentabilidade em Organizações (SEO) também para cada empresa. As avaliações de Grau de Complexidade Cognitiva (GCC) e Sustentabilidade em Organizações (SEO) foram realizadas sob as óticas do modelo de Criação de Valor Sustentável (CVS) e do ISE respectivamente. Portanto, cada empresa $x$ foi associada a um par de medidas $\left(M G C C_{x}, M S E O_{x}\right)$ do Grau de Complexidade Cognitiva (GCC) e da Sustentabilidade em Organizações (SEO), sendo que essas medidas foram propostas neste artigo. É importante destacar que os mapas cognitivos associados a cada empresa, denominados mapas de Criação de Valor Sustentável ou mapas CVS, contribuíram para avaliar o GCC e identificar quais eram as estratégias, as práticas e as ações adotadas pelas empresas em seus ambientes interno e externo, e nos seus planos do presente e para o futuro; e os questionários do ISE contribuíram para avaliar a SEO e verificar o desempenho de cada empresa em relação às suas dimensões, critérios e indicadores da sustentabilidade.

Resultados de análises sobre os mapas CVS mostraram que as empresas estudadas concentram maior atenção em práticas e ações relacionadas à estratégia de Gerenciamento de Produto, seguida de Combate à Poluição, Tecnologia Limpa e Base da Pirâmide. Esses resultados corroboram com outras publicações (Hart, 2005; Hart \& Milstein, 2003), que explicam que as organizações têm maior facilidade de trabalhar no campo inferior do modelo de Criação de Valor Sustentável (CVS), o qual comporta as duas estratégias principais do presente, ou seja, Gerenciamento de Produto e Combate à Poluição. Resultados de análises sobre os questionários do ISE das empresas mostraram que a dimensão com melhor desempenho para o conjunto de casos é a Natureza do Produto, e que a dimensão com menor desempenho se refere a Mudanças Climáticas. Observou-se, durante as análises, que o questionário do ISE não apresenta dimensões, critérios e indicadores diretamente relacionados à estratégia de Tecnologia Limpa pertencente ao modelo de CVS. Entretanto, com base nos relatórios de sustentabilidade, foi possível verificar a adoção parcial da estratégia Tecnologia Limpa pelas empresas e explicitar seus construtos e links associados aos mapas de CVS de cada organização do estudo de casos múltiplos.

Resultados de uma análise comparativa entre os valores encontrados para as medidas normalizadas de grau de complexidade cognitiva $\left(M G C C_{x}{ }_{x}\right)$ e sustentabilidade em organizações $\left(M S E O_{x)}\right.$ indicaram a existência de uma relação com tendência positiva entre GCC e SEO. Ao constatar a existência dessa relação e da importância da adoção de estratégias e dimensões relacionadas à sustentabilidade, este trabalho recomenda utilizar os valores de $M G C C^{\prime}$ e $M S E O$ para estudos de benchmarking em sustentabilidade de empresas e seus setores.

Os resultados apresentados mostram que as empresas estudadas possuem estratégias e dimensões da sustentabilidade. Contudo, algumas empresas atendem mais ou menos estratégias e dimensões da sustentabilidade que outras empresas. Sob esta perspectiva, pode-se afirmar que algumas empresas são mais sustentáveis que outras. Aquelas mais sustentáveis satisfazem mais critérios que as outras. Dessa forma, conclui-se que o conceito de sustentabilidade em organizações pode ser caracterizado como um conjunto nebuloso (Zadeh, 1965), que contém elementos (empresas) com diferentes graus de compatibilidade ou pertinência no conjunto de empresas sustentáveis.

Entre as seis empresas estudadas neste artigo, duas estão incluídas no Global 100, que constitui uma lista de classificação das 100 empresas mais sustentáveis no mundo, sendo elas a Natura, classificada em segundo lugar, e o Banco do Brasil, classificado no centésimo lugar. Essa informação corrobora com os resultados deste artigo, uma vez que a Natura obteve um valor de $M S E O$ um pouco acima do valor obtido para o Banco do Brasil.

É importante observar que as três empresas que apresentaram maiores valores de $M G C C^{\prime} \mathrm{e}$ $M S E O$, sendo elas Banco do Brasil, Eletropaulo e Natura, compõem o ISE desde a sua criação, em 2005. Esse fato pode ser usado para questionar se o GCC e o SEO associados a cada uma dessas empresas foram desenvolvidos em virtude das suas participações no ISE por um tempo mais prolongado e contínuo quando comparado às demais, uma vez que o ISE exige das empresas uma evolução de seus indicadores para que possam permanecer na sua composição. 
Buscando-se aprofundar o conhecimento sobre o tema investigado, este artigo sugere algumas pesquisas futuras, como: (a) a realização de estudos contingenciais entre cognição e sustentabilidade em organizações; (b) a utilização de levantamentos com uma população mais expressiva de empresas que possibilitem encontrar correlações estatísticas entre as categorias pesquisadas, permitindo a generalização de resultados; (c) a realização de estudos longitudinais que possam mostrar a evolução das categorias estudadas; e (d) a investigação da relação entre adoção de estratégias da sustentabilidade e desempenho financeiro-econômico de empresas.

\section{Referências}

Aguinis, H., \& Glavas, A. (2012). What we know and don't know about corporate social responsibility: a review and research agenda. Journal of Management, 38(4), 932-968. doi: $10.1177 / 0149206311436079$

Barbieri, J. C., \& Cajazeira, J. E. R. (2012). Responsabilidade social empresarial e empresa sustentável: da teoria a prática. São Paulo: Saraiva.

Barbieri, J. C., Vasconcelos, I. F. G., Andreassi, T., \& Vasconcelos, F. C. (2010). Inovação e sustentabilidade: novos modelos e proposições. Revista de Administração de Empresas, 50(2), 146-154. doi: 10.1590/S0034-75902010000200002

Bardin, L. (2009). Análise de conteúdo (Edição revista e atualizada). Lisboa: Edições 70.

Barney, J. B. (1991). Firm resources and sustained competitive advantage. Journal of Management, 17(1), 99-120. doi: 10.1177/014920639101700108

Barney, J. B. (1996). The resource-based theory of the firm. Organization Science, 7(5), 469. doi: 10.1287/orsc.7.5.469

Bastos, A. V. (2002). Mapas cognitivos e a pesquisa organizacional: explorando aspectos metodológicos (Especial Natal). Estudos de Psicologia, (7), 65-77. doi: 10.1590/S1413294X2002000300008

Beeby, A., \& Brennan, A. (2007). First ecology: ecological principles and environmental issues. Oxford: Oxford University Press.

Bertalanffy, L. Von (1968). General system theory: foundations, development, and applications. New York: Allen Lane.

BM\&FBOVESPA. (n.d.). Novo valor: sustentabilidade nas empresas - Como começar, quem envolver $e$ que priorizar. Recuperado de http://www.bmfbovespa.com.br/empresas/download/guia-de-sustentabilidade.pdf

BM\&FBOVESPA. (2011, novembro 25). BM\&FBOVESPA divulga nova carteira do Índice de Sustentabilidade Empresarial para 2012 . Recuperado de http://www.bmfbovespa.com.br/Indices/download/Nova-carteira-ISE-2012.pdf

BM\&FBOVESPA. (2013). Carteiras Índice de Sustentabilidade Empresarial - ISE (Abertura). Recuperado de http://www.bmfbovespa.com.br/Indices/download/Evolutivo-das-carteirasISE.pdf

Boulding, K. E. (1956). General systems theory: the skeleton of science. Management Science, 2(3), 197-208. doi: 10.1287/mnsc.2.3.197

Bunge, M., \& Ardila, R. (1987). Philosophy of psychology. New York: Springer-Verlag. 
Calori, R., Johnson, G., \& Sarnin, P. (1994). CEO's cognitive maps and the scope of the organization. Strategic Management Journal, 15(6), 437-457. doi: 10.1002/smj.4250150603

Centro de Estudos em Sustentabilidade. (2012a). Critérios e pesos: ISE 2012-2013. Recuperado de https://www.isebvmf.com.br/arquivos/37/ISE_2012-CriteriosePesos.pdf

Centro de Estudos em Sustentabilidade. (2012b). O valor do ISE. Principais estudos e a perspectiva dos investidores. São Paulo: BM\&FBOVESPA. Recuperado de http://www.bmfbovespa.com.br/Indices/download/O-Valor-do-ISE.pdf

Dahlsrud, A. (2008). How corporate social responsibility is defined: an analysis of 37 definitions. Corporate Social Responsibility and Environmental Management, 15(1), 1-13. doi: $10.1002 / \mathrm{csr} .132$

Easterlin, R. A. (2000). The worldwide standard of living since 1800. The Journal of Economic Perspectives, 14(1), 7-26. doi: 10.1257/jep.14.1.7

Eden, C. (1992). On the nature of cognitive maps. Journal of Management Studies, 29(3), 261-265. doi: 10.1111/j.1467-6486.1992.tb00664.x

Eden, C. (2004). Analyzing cognitive maps to help structure issues or problems. European Journal of Operational Research, 159(3), 673-686. doi: 10.1016/S0377-2217(03)00431-4

Ehrlich, P. R. (1968). The population bomb. New York: Ballantine Books.

Eisenhardt, K. M. (1989). Building theories from case study research. Academy of Management Review, 14(4), 532-550.

Elkington, J. (1994). Towards the sustainable corporation: win-win-win business strategies for sustainable development. California Management Review, 36(2), 90-100.

Elkington, J. (1997). Cannibals with Forks: the triple bottom line of 21 st century business. Oxford: Capstone.

Elkington, J. (2004). Enter the triple bottom line. In A. Henriques \& J. Richardson (Eds.), The triple bottom line, does it all add up? (pp. 1-16). London: Earthscan.

Fiol, C. M., \& Huff, A. S. (1992). Maps for managers: where are we? Where do we go from here? Journal of Management Studies, 29(3), 267-285. doi: 10.1111/j.1467-6486.1992.tb00665.x

Garriga, E., \& Melé, D. (2004). Corporate social responsibility theories: mapping the territory. Journal of Business Ethics, 53(1-2), 51-71. doi: 10.1023/B:BUSI.0000039399.90587.34

Gladwin, T. N., Kennelly, J. J., \& Krause, T. (1995). Shifting paradigms for sustainable development: implications for management theory and research. The Academy of Management Review, 20(4), 874-907. doi: $10.2307 / 258959$

Goodland, R. (1995). The concept of environmental sustainability. Annual Review of Ecology and Systematics, 26, 1-24. doi: 10.1146/annurev.es.26.110195.000245

Gordon, R. J. (2000). Does the new economy measure up to the great inventions of the past? The Journal of Economic Perspectives, 14(4), 49-74. doi: 10.1257/jep.14.4.49

Hall, A. D., \& Fagen, R. E. (1956). Definition of system. In W. Buckley (Ed.), Modern systems research for the behavioral scientist (pp. 81-92). Chicago: Aldine Publishing Company.

Hart, S. L. (1995). A natural-resource-based view of the firm. The Academy of Management Review, 20(4), 986-1014. doi: 10.2307/258963 
Hart, S. L. (1997). Beyond greening: strategies for a sustainable world. Harvard Business Review, 75(1), 66-76.

Hart, S. L. (2005). Capitalism at the crossroads: the unlimited business opportunities in solving the world's most difficult problems. Upper Saddle River, NJ: Wharton School Publishing.

Hart, S. L., \& Christensen, C. M. (2002). The great leap: driving innovation from the base of the pyramid. Sloan Management Review, 44(1), 51-56.

Hart, S. L., \& Dowell, G. (2011). Invited editorial: a natural-resource-based view of the firm: fifteen years after. Journal of Management, 37(5), 1464-1479. doi: 10.1177/0149206310390219

Hart, S. L., \& Milstein, M. B. (2003). Creating sustainable value. Academy of Management Executive, 17(2), 56-67.

Hawken, P., Lovins, A., \& Lovines, L. H. (1999). Natural capitalism: creating the next industrial revolution. Boston: Little, Brown and Company.

Johnson, D. G. (2000). Population, food, and knowledge. The American Economic Review, 90(1), 114. doi: 10.1257/aer.90.1.1

Kandachar, P., \& Halme, M. (2008). Sustainability challenges and solutions at the base of the pyramid - business, technology and the poor. Sheffield-UK: Greenleaf.

Kurapatskie, B., \& Darnall, N. (2013). Which corporate sustainability activities are associated with greater financial payoffs? Business Strategy and the Environment, 22(1), 49-61. doi: $10.1002 / b s e .1735$

Maia, A. G., \& Pires, P. S. dos (2011). Uma compreensão da sustentabilidade por meio dos níveis de complexidade das decisões organizacionais. Revista de Administração Mackenzie, 12(3), $177-$ 206. doi: 10.1590/S1678-69712011000300008

Marcondes, A. W., \& Bacarji, C. D. (2010). ISE - Sustentabilidade no mercado de capitais. São Paulo: Report Editora.

Meadows, D. H., Meadows, D. L., Randers, J., \& Behrens, W.W., III (1972). The limits to growth: a report for the Club of Rome's project on the predicament of mankind. New York: Universe Books.

Mozzato, A. R., \& Grzybovski, D. (2011). Análise de conteúdo como técnica de análise de dados qualitativos no campo da administração: potencial e desafios (Documentos e Debates). Revista de Administração Contemporânea, 15(4), 731-747. Recuperado de http://www.scielo.br/pdf/rac/v15n4/a10v15n4.pdf. doi: 10.1590/S1415-65552011000400010

Nidumolu, R., Prahalad, C. K., \& Rangaswami, M. R. (2009). Why sustainability is now the key driver of innovation? Harvard Business Review, 87(9), 56-65.

Nobre, F. S., Tobias, A. M., \& Walker, D. S. (2009). Organizational and technological implications of cognitive machines: designing future information management systems. New York: IGI Global.

Nobre, F. S., Tobias, A. M., \& Walker, D. (2010). A new contingency view of the organization: managing complexity and uncertainty through cognition. Brazilian Administration Review, 7(4), 379-396. Recuperado de http://www.scielo.br/pdf/bar/v7n4/05.pdf. doi: 10.1590/S180776922010000400005

Nobre, F. S., Tobias, A. M., \& Walker, D. S. (2011). Uma visão da empresa baseada em habilidades: contextos estratégicos e contingenciais. Revista de Administração Contemporânea, 15(3), 413- 
432. Recuperado de http://www.scielo.br/pdf/rac/v15n3/v15n3a04.pdf. doi: 10.1590/S141565552011000300004

Nobre, F. S., \& Walker, D. (2011b). A dynamic ability-based view of the organization. International Journal of Knowledge Management, 7(2), 86-101.

Nobre, F. S., \& Walker, D. (2011a). An ability-based view of the organization: strategic-resource and contingency domains. The Learning Organization, 18(4), 333-345. doi: $10.1108 / 09696471111132531$

Nobre, F. S., Walker, D., \& Harris, R. (2012). Technological, managerial and organizational core competencies: dynamic innovation and sustainable development. New York: IGI Global.

Organização das Nações Unidas para a Educação, a Ciência e a Cultura. (2005). Década da educação das nações unidas para um desenvolvimento sustentável, 2005-2014: documento final do esquema internacional de implementação. Brasília: Autor.

Porac, J. F., \& Thomas, H. (2002). Managing cognition and strategy: issues, trends and future directions. In A. M. Pettigrew, H. Thomas, \& R. Whittington (Eds.), Handbook of strategy and management (pp. 165-181). London: Sage.

Prahalad, C. K. (2004). The fortune at the bottom of the pyramid: eradicating poverty through profits. Upper Saddle River, NJ, USA: Wharton School Publishing.

Prahalad, C. K., \& Hart S. L. (2002). The fortune at the bottom of the pyramid. Strategy + Business, (26), 2-14.

Richter, M. N. (1982). Technology and social complexity. New York: State University of New York.

Rogers, P. P., Jalal, K. F., \& Boyd, J. A. (2008). An introduction to sustainable development. London: Earthscam.

Sachs, I. (2000). Caminhos para o desenvolvimento sustentável. Rio de Janeiro: Garamond.

Simon, H. A. (1996). The sciences of the artificial (3rd ed.). Cambridge: MIT Press.

Teece, D. J., Pisano, G., \& Shuen, A. (1997). Dynamic capabilities and strategic management. Strategic Management Journal, 18(7), 509-533. doi: 10.1002/(SICI)10970266(199708)18:7<509::AID-SMJ882>3.0.CO;2-Z

Thompson, L. J. (2010). The global moral compass for business leaders. Journal of Business Ethics, 93(1), 15-32. doi: 10.1007/s10551-010-0624-9

United Nations Environment Programme. (2012). GEO5 Global environment outlook: environment for the future we want. Malta: Progress Press Ltd.

United Nations Global Compact. (2011). Corporate sustainability in the world economy. New York: UN Global Compact Office.

United Nations World Commission on Environment and Development. (1987). Our common future. Oxford: Oxford University Press.

Vivien, F.-D. (2011). Economia e ecologia. São Paulo: SENAC.

Walsh, J. P. (1995). Managerial and organizational cognition: notes from a trip down memory lane. Organization Science, 6(3), 280-321. doi: 10.1287/orsc.6.3.280

Yin, R. K. (2005). Estudo de caso: planejamento e métodos (3a ed., D. Grassi, Trad.). Porto Alegre: Bookman. (Obra original publicada em 1984).

Zadeh, L. A. (1965). Fuzzy sets. Information and Control, 8(3), 338-353.

RAC, Rio de Janeiro, v. 17, n. 4, art. 6, pp. 499-517, Jul./Ago. 2013

www.anpad.org.br/rac $((\mathrm{coc})$ 